\title{
High-resolution direction-of-arrival estimation using Khatri-Rao product and spatial sparsity of sources
}

\author{
Shanshan Ding ${ }^{1, a}$, Ningning Tong ${ }^{2, b}$ and Qichao $\mathrm{Ge}^{3, \mathrm{c}}$ \\ ${ }^{1}$ Air Force Engineering University, Xi'an, Shaanxi, China \\ ${ }^{2}$ Air Force Engineering University, Xi'an, Shaanxi, China \\ ${ }^{3}$ Air Force Engineering University, Xi'an, Shaanxi, China \\ adingshanshan001@hotmail.com, ㅎ2647005034@qq.com, geqichao927@163.com
}

\begin{abstract}
Keywords: DOA estimation; K-R product; spatial sparsity;
Abstract. Algorithms based on Khatri-Rao (K-R) product improve the degree of freedom (DOF) of virtual arrays and thus more source angles can be estimated. However, the estimation accuracy is not satisfying. In this paper, a method considering spatial sparsity (SS) of sources in K-R subspace is proposed to estimate direction-of-arrival (DOA) angle. The spurious signal of the virtual array is obtained in K-R subspace and then the DOA angle estimation is finally equal to the solution of a convex optimization problem. In the proposed K-R SS method, high estimation accuracy is achieved by taking advantage of the cross-covariance matrix information. Simulation results demonstrate the effectiveness of the proposed method.
\end{abstract}

\section{Introduction}

A number of high-resolution techniques for direction-of-arrival (DOA) estimation in radar, sonar and wireless communications have been proposed in recent years $[1,2,3]$. However, the majority of these techniques are confined to detect lesser sources than sensors, i.e. the maximum number of sources which can be resolved with a uniform linear array (ULA) of $N$ sensors is $N-1$. To solve this problem, virtual array is produced by adopting non-uniform array and special algorithms to increase the degrees of freedom (DOF) of array. The existing two typical algorithms to produce virtual array is fourth-order cumulant [4] and K-R product [5,6,7,8]. Compared with algorithms using fourth-order cumulant, algorithms based on K-R product are more computational efficient and fewer snapshots is required. However, there are still some problems about K-R product based algorithms.

In K-R subspace, by vectoring the covariance matrix of the original echo signal, the equivalent echo signal of virtual array is obtained, i.e. the spurious signal. Although the spurious signal has a similar form of the original echo signal and consists of signal component and noise component as well, the difference between the two signals is not only the dimension of steering vector, but also the signal-to-signal coherence and signal-to-noise coherence. Moreover, the noise component of spurious signal is obviously not Gaussian white, which breaks the basic assumption of most DOA estimation methods. One solution of the foresaid problems proposed in [7] is called K-R MUSIC method, in which spatial smoothing algorithm is utilized to work out the coherence, and then the traditional multiple signal classification (MUSIC) algorithm can be used to estimate DOA angle. The limit of K-R MUSIC is that the number of sources is required in advance and the expected estimation accuracy is barely achieved. The other solution proposed in [9] is called K-R CS method, in which compressed sensing (CS) theory is used to avoid the influence caused by coherence and DOA angle estimation is obtained without knowing the number of sources beforehand. Whereas, false peaks make a bad influence on K-R CS and even let it fail in a low SNR condition.

In order to obtain high estimation accuracy and a stable performance in bad electromagnetic environment, a DOA estimation method in K-R subspace is proposed based on spatial sparsity of sources in this paper. To decorrelate signal-to-signal coherence better, the covariance matrix information and cross-covariance matrix information of virtual sub-arrays are fully utilized via weighted summation. After that, the unknown noise and signal-to-noise coherence can be solved 
through the weighted matrix determined in the light of spatial representation theory. It is noted that the proposed K-R SS method does not require the prior information of source number.

\section{Signal model}

Consider a $N$ linear array impinged by $D$ narrowband sources from directions $\left\{\theta_{i}, i=1,2, \ldots, D\right\}$ with powers $\left\{\sigma_{i}^{2}, i=1,2, \ldots, D\right\}$, the echo signal is

$$
\boldsymbol{X}(t)=\boldsymbol{A S}(t)+\boldsymbol{N}(t) \quad t=1,2, \ldots, M .
$$

where $\boldsymbol{X}(t)=\left[\boldsymbol{x}_{1}(t), \ldots, \boldsymbol{x}_{N}(t)\right]^{\mathrm{T}} \in \mathrm{f}^{N \times 1}$ denotes the echo signal of the $t$ th snapshot. $\left(g^{\mathrm{T}}\right.$ means transpose operator and $M$ is the number of snapshots. The array manifold matrix $\boldsymbol{A}=\left[\boldsymbol{a}\left(\theta_{1}\right), \ldots, \boldsymbol{a}\left(\theta_{D}\right)\right] \in \mathrm{f}^{N \times D} \quad$ consists $\quad$ of $\quad D \quad$ steering vectors $\boldsymbol{a}\left(\theta_{i}\right)=\left[\exp \left(\mathrm{j} 2 \pi x_{1} \sin \theta_{i} / \lambda\right), \ldots, \exp \left(\mathrm{j} 2 \pi x_{n} \sin \theta_{i} / \lambda\right)\right]^{\mathrm{T}}, n=1, \ldots, N$, where $x_{n}$ denotes the position of $n$th sensor and $\lambda$ denotes wave length. $S(t)=\left[s_{1}(t), \ldots, s_{D}(t)\right]^{\mathrm{T}} \in \mathrm{f}^{D \times 1}$ is the zero mean signal vector and $N(t) \in €^{N \times 1}$ is Gaussian white noise with power $\sigma_{n}{ }^{2}$. The noise covariance matrix is written as $\boldsymbol{R}_{N N}=\sigma_{n}{ }^{2} \boldsymbol{I}_{N} \in \mathrm{f}^{N \times N}$, where $\left(\mathrm{g}^{\mathrm{H}}\right.$ denotes conjugate transpose operator and $\boldsymbol{I}_{N}$ is the unit matrix of size $N \times N$.

Therefore, the covariance matrix of echo signal is

$$
\boldsymbol{R}_{\boldsymbol{X} \boldsymbol{X}}=\mathrm{E}\left\{\boldsymbol{X}(t) \boldsymbol{X}(t)^{\mathrm{H}}\right\}=\boldsymbol{A} \boldsymbol{R}_{S S} \boldsymbol{A}^{\mathrm{H}}+\boldsymbol{R}_{\boldsymbol{N}}=\boldsymbol{A} \boldsymbol{p} \boldsymbol{A}^{\mathrm{H}}+\sigma_{\mathrm{n}}{ }^{2} \boldsymbol{I}_{\boldsymbol{N}} \cdot
$$

where $\boldsymbol{R}_{S S}=\operatorname{diag}(\boldsymbol{p})$ is the covariance matrix of signal, $\boldsymbol{p}=\left[{\sigma_{1}}_{1}^{2},{\sigma_{2}}^{2}, \mathrm{~L},{\sigma_{D}}^{2}\right]$ is signal power vector and denotes a $D \times D$ diagonal matrix with vector $p \in{ }^{\circ}{ }^{D}$ on the main diagonal.

Given $\boldsymbol{A} \in \mathrm{f}^{n \times k}$ and $\boldsymbol{B} \in \mathrm{f}^{m \times k}$, the K-R product is then denoted by

$$
\boldsymbol{A} \circ \boldsymbol{B}=\left[\boldsymbol{a}_{\boldsymbol{l}} \otimes \boldsymbol{b}_{1}, \ldots, \boldsymbol{a}_{f} \otimes \boldsymbol{b}_{f}\right] \in \mathfrak{f}^{n m \times k} .
$$

where $\otimes$ is the Kronecker product. A significant property of K-R product proved in [6] is

$$
\operatorname{vec}\left(\boldsymbol{A D} \boldsymbol{B}^{\mathrm{H}}\right)=\left(\boldsymbol{B}^{*} \text { OA }\right) \boldsymbol{d} \text {. }
$$

where $\boldsymbol{D}=\operatorname{diag}(\boldsymbol{d})$ and $\left(g^{*}\right.$ denotes conjugate operator.

\section{K-R SS DOA Estimation Method}

Based on the property of K-R product, the Eq. 2 can be rewritten as

$$
\boldsymbol{y}=\operatorname{vec}\left(\boldsymbol{R}_{\boldsymbol{X X}}\right)=\operatorname{vec}\left(\boldsymbol{A} \boldsymbol{R}_{S S} \boldsymbol{A}^{\mathrm{H}}\right)+\operatorname{vec}\left(\boldsymbol{R}_{N N}\right)=\left(\boldsymbol{A}^{*} \mathrm{O} \boldsymbol{A}\right) \boldsymbol{p}+\sigma_{\mathrm{n}}{ }^{2} \operatorname{vec}\left(\mathbf{I}_{\mathrm{N}}\right)
$$

It is obvious that $\boldsymbol{A}^{*} \mathrm{O} \boldsymbol{A} \in \mathrm{f}^{N^{2} \times D}$ has the similar form with the manifold matrix $\boldsymbol{A}$. However, the row of $\boldsymbol{A}^{*} \mathrm{OA}$, which is connected with sensor position of virtual ULA obtained in K-R subspace, is out of order. By rearranging $\boldsymbol{A}^{*} \mathrm{OA}$ in accordance with the sensor positions of virtual ULA and averaging the repeated rows, the spurious signal of virtual ULA is as follows

$$
z=\boldsymbol{A}_{K R} \boldsymbol{p}+\sigma_{n}^{2} \boldsymbol{I}^{\prime} \text {. }
$$

where $\boldsymbol{A}_{K R}$ is the manifold matrix of virtual ULA and $\boldsymbol{I}^{\prime}$ is the noise.

In order to maximize the DOF of array to improve estimation performance, a two level nested passive array with $N$ sensors provided in [7] is adopted and a virtual ULA with $N v$ sensors in $d$ inner space is then obtained in K-R subspace, where $N v=N^{2} / 2+N-1$. Thus, the dimension of $z$ and $\boldsymbol{A}_{\boldsymbol{K} \boldsymbol{R}}$ are $N v \times 1$ and $N v \times D$, respectively. $\boldsymbol{A}_{\boldsymbol{K} \boldsymbol{R}}$ is expressed as follows 


$$
\boldsymbol{A}_{K R}=\left(\begin{array}{cccc}
1 & 1 & \mathrm{~K} & 1 \\
v_{1} & v_{2} & \mathrm{~K} & v_{D} \\
\mathrm{M} & \mathrm{M} & \mathrm{O} & \mathrm{M} \\
v_{1}^{N \nu-1} & v_{2}^{N v-1} & \mathrm{~K} & v_{D}^{N v-1}
\end{array}\right) .
$$

where $v_{i}=\exp \left(\mathrm{j} 2 \pi d \sin \theta_{i} / \lambda\right)$. The noise $\boldsymbol{I}^{\prime} \in{ }^{\circ{ }^{N \times 1}}$ is a vector of all zeros except a 1 at the $N f$ th position, where $N f=\left(N^{2} / 4+N / 2\right)$.

Since the signal-to-signal coherence makes the covariance matrix of $z$ a singular matrix, spatial smoothing algorithm is applied to recovery the rank of covariance matrix. In this case, the virtual array is divided into $N f$ sub-arrays and each one has $N f$ virtual sensors.

The spurious signal of $j$ th sub-array, which corresponds to the $(N f-j+1)$ th to $(N v-j+1)$ th rows of $z$, is thus denoted as

$$
\boldsymbol{z}_{j}=\boldsymbol{A}_{\boldsymbol{l}} \boldsymbol{\Phi}^{j-1} \boldsymbol{p}+\sigma_{n}{ }^{2} \boldsymbol{I}^{\prime \prime}, \quad 1 \leq j \leq N f .
$$

where $\boldsymbol{A}_{\boldsymbol{I}}$ is the manifold matrix of the first sub-array, $\boldsymbol{\Phi}=\operatorname{diag}\left(v_{l}, \mathrm{~L}, v_{D}\right)$ and $\boldsymbol{I}^{\prime \prime} \in{ }^{\circ f \times 1}$ is a vector of all zeros except a 1 at the $j$ th position.

Thus, The full-rank covariance matrix of sub-arrays is

$$
\boldsymbol{R}_{\text {sum }}{ }^{\prime}=\sum_{j=1}^{N f} \boldsymbol{F}_{j} \boldsymbol{R}_{z z} \boldsymbol{F}_{j} .
$$

$$
\text { where } \boldsymbol{R}_{z z}=\mathrm{E}\left\{z z^{\mathrm{H}}\right\} \in \mathrm{f}^{N_{v} \times N_{v}} \text { is the covariance matrix of } \boldsymbol{z} \text { and } \boldsymbol{F}_{\boldsymbol{j}}=\left\lfloor\boldsymbol{\boldsymbol { O }}_{N f \times(j-1)}\left|\boldsymbol{I}_{N f}\right| \boldsymbol{\boldsymbol { O }}_{N f \times(N f-j)}\right\rfloor \text {. }
$$

Furthermore, for better performance, the cross covariance matrix information of sub-arrays is added by weighted summation, and the full-rank covariance matrix of sub-arrays is rewritten by

$$
\boldsymbol{R}_{\text {sum }}=\sum_{j=1}^{\mathrm{Nf}} \sum_{k=1}^{\mathrm{Nf}} \boldsymbol{F}_{j} \boldsymbol{R}_{z z} \boldsymbol{F}_{\boldsymbol{k}} w_{j k} .
$$

where $w_{j k}$ is the $(j, k)$ th element of the weighted matrix $\boldsymbol{W}$.

It should be noted that the matrix $\boldsymbol{R}_{\text {sum }}$ is made of two components: signal component $\boldsymbol{R}_{S}=\boldsymbol{A}_{\boldsymbol{I}} \boldsymbol{\Lambda} \boldsymbol{A}_{\boldsymbol{I}}{ }^{\mathrm{H}} \boldsymbol{W} \boldsymbol{A}_{\boldsymbol{I}} \boldsymbol{\Lambda} \boldsymbol{A}_{\boldsymbol{I}}{ }^{\mathrm{H}}$ and noise component $\boldsymbol{R}_{\boldsymbol{N}}=\sigma_{n}^{4} \boldsymbol{W}+\sigma_{n}^{2} \boldsymbol{A}_{\boldsymbol{I}} \boldsymbol{\Lambda} \boldsymbol{A}_{\boldsymbol{I}}{ }^{\mathrm{H}} \boldsymbol{W}+\sigma_{n}^{2} \boldsymbol{W}^{\mathrm{H}} \boldsymbol{A}_{\boldsymbol{I}} \boldsymbol{\Lambda} \boldsymbol{A}_{\boldsymbol{I}}{ }^{\mathrm{H}}$, where $\boldsymbol{\Lambda}=\boldsymbol{R}_{s s}$ contains the sources information on main diagonal.

By utilizing the spatial sparsity of sources, $\boldsymbol{W}$ is constructed as

$$
\boldsymbol{W}=\left(\boldsymbol{B}_{\boldsymbol{K} \boldsymbol{R}} \hat{\boldsymbol{A}}_{\boldsymbol{K} \boldsymbol{R}}^{\mathrm{H}}\right)^{-1}=\left(\boldsymbol{B}_{\boldsymbol{K} \boldsymbol{R}} \operatorname{diag}(\hat{\boldsymbol{p}}) \boldsymbol{B}_{\boldsymbol{K} \boldsymbol{R}}{ }^{\mathrm{H}}\right)^{-1} \text {. }
$$

where the signal vector $\hat{\boldsymbol{p}} \in \mathrm{f}^{P}$ is $K$ - sparse using the direction grid $P$, i.e. $\hat{\boldsymbol{p}}$ has at most $K=P$ non-zero entries (let $K=D$ in this paper). $\hat{\boldsymbol{\Lambda}}=\operatorname{diag}(\hat{\boldsymbol{p}})$ is a diagonal matrix with the sources information contained by elements on main diagonal in $K$ - sparse, and $\boldsymbol{B}_{\boldsymbol{K} \boldsymbol{R}} \in \mathbf{f}^{N f \times P}$ is the over-complete dictionary matrix for virtual sub-arrays.

Therefore, the DOA angels estimation, which is thus equivalent to the recovery of $\hat{\boldsymbol{p}}$ from $\hat{\boldsymbol{R}}_{S}=\boldsymbol{B}_{K \boldsymbol{R}} \hat{\boldsymbol{A}}_{\boldsymbol{K} \boldsymbol{R}}^{\mathrm{H}}$, is represented by

$$
\overline{\boldsymbol{p}}=\underset{\hat{\boldsymbol{p}} \in f^{P}}{\arg \min }\left\|\boldsymbol{R}_{\text {sum }}-\hat{\boldsymbol{R}}_{S}\right\|_{2} \quad \text { s.t. }\|\hat{\boldsymbol{p}}\|_{0} \leq K .
$$

where $\|\hat{\boldsymbol{p}}\|_{0}:=\left|\left\{i: \hat{p}_{i} \neq 0\right\}\right|$ is the $l_{0}$ - norm of $\hat{\boldsymbol{p}}$.

However, Eq. 11 is combinatorial and computationally intractable except for small problems, and one commonly used approach is to solve a closely related $l_{1}$ - minimization problem 


$$
\overline{\boldsymbol{p}}=\underset{\hat{\boldsymbol{p}} \in \mathrm{f}^{p}}{\arg \min }\left\|\boldsymbol{R}_{\text {sum }}-\hat{\boldsymbol{R}}_{S}\right\|_{2} \quad \text { s.t. }\|\hat{\boldsymbol{p}}\|_{1} \leq K .
$$

where $\|\hat{\boldsymbol{p}}\|_{1}:=\sum_{i}\left|\hat{p}_{i}\right|$.

Eq. 12 is thus a convex optimization problem [10] and can be rewritten as

$$
\overline{\boldsymbol{p}}=\underset{\hat{p} \in \mathrm{f}^{p}}{\arg \min } \frac{1}{2}\left\|\boldsymbol{R}_{\text {sum }}-\hat{\boldsymbol{R}}_{S}\right\|_{2}^{2}+\mu\|\hat{\boldsymbol{p}}\|_{1} \text {. }
$$

\section{Simulations}

In this section, the effectiveness of our method is tested. Assume that a 6 sensors two level nested passive array $(N=6)$ with10 uncorrelated narrowband sources $(D=10)$ impinging on it from directions of arrival $\left\{-55^{\circ},-40^{\circ},-30^{\circ},-15^{\circ},-5^{\circ}, 10^{\circ}, 15^{\circ}, 30^{\circ}, 45^{\circ}, 60^{\circ}\right\}$, with all equal powers. In each simulation 1024 snapshots and 1000 Monte-Carlo simulations are used.

In the first simulation, the RMSEs against SNR are plotted in Fig. 1, where the SNR varies from $-10 \mathrm{~dB}$ to $20 \mathrm{~dB}$. The RMSE of angle estimation is defined as RMSE $=\sqrt{\frac{1}{\mathrm{D}} \sum_{i=1}^{\mathrm{D}} \mathrm{E}\left\{\left(\hat{\theta_{i}}-\theta_{i}\right)^{2}\right\}}$, where $\theta_{i}$ is the real DOA angle and $\hat{\theta_{i}}$ is the estimated one for the $i$ th source. It can be seen from Fig. 1 that K-R SS method proposed in this paper outperforms K-R MUSIC method and K-R CS method, which is due to the added cross-covariance matrix information. K-R SS method has higher estimation accuracy than the others, and it also has better robustness than K-R CS based method even in low SNR condition.

In the second simulation, the probability of successful estimation is taken into consideration.

Because of the high RMSE of K-R MUSIC method showed in Fig. 1, only the successful probability of $\mathrm{K}-\mathrm{R}$ CS and K-R SS method are compared. If the array elements spacing is longer than a half wave length, false peaks may appear. A successful estimation is thus defined as an estimation without false peaks and the probability of success is the ratio of the successful estimation to the whole Monte-Carlo simulations. As shown in Fig. 2, the probability of success with K-R SS method is compared with that of K-R CS method when the SNR varies from $-10 \mathrm{~dB}$ to $0 \mathrm{~dB}$. Obviously, the success probability of K-R SS method is much higher than that of K-R CS method. Finally, the success probability of K-R CS method catches up with that of K-R SS method at 0dB. It indicates that K-R SS method outperforms the K-R CS method in low SNR conditions.

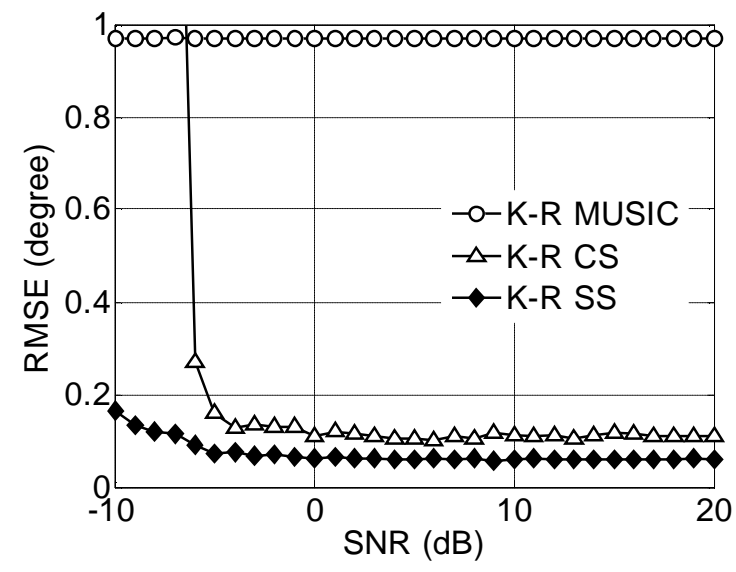

Fig. 1. RMSE versus SNR

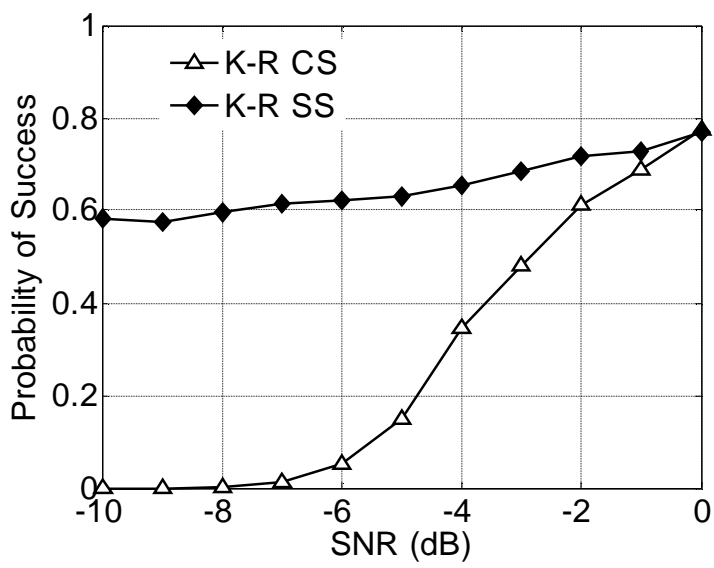

Fig. 2. Probability of Success versus SNR

\section{Conclusions}

We have developed a novel DOA estimation method, which called K-R SS method in this paper. On the basis of having the capability to detect more sources than sensors via K-R product, a higher estimation accuracy is achieved by spatial sparsity of sources because of the added cross-covariance 
matrix information of virtual sub-arrays. Simulation results imply that the proposed method outperforms the previous ones.

\section{References}

[1] T. E. Tuncer, B. Friedlander: Classical and modern direction-of-arrival estimation,( Elsevier, 2009, USA).

[2] P. S. Naidu:Sensor array signal processing.( CRC Press, USA, 2010).

[3] H. L. Van Trees: Detection, estimation, modulation theory, optimum array processing (Wiley-Interscience, USA,2004).

[4] P. Chevalier, L. Albera, A. Ferreol and P. Comon. IEEE Trans. on Signal Processing Vol.53(2005), p.1254-1271

[5] W. K. Ma, T. H. Hsieh and C. Y. Chi. Proc. IEEE Int. Conf. Acoust. Speech Signal Process. (ICASSP), 2009, p.2165-2168

[6] W. K. Ma, T. H. Hsieh and C. Y. Chi. IEEE Trans. on Signal Processing Vol.58(2010), p. $2168-2180$

[7] P Pal, P. Vaidyanathan. IEEE Trans. on Signal Processing Vol.58(2010), p. 4167-4181

[8] S. Li, W. He, X. Yang, M. Bao and Y Wang. Journal of Central South University. Vol.21(2014), p.2743-2750

[9] N. Hu, Z. Ye, X. Xu and M. Bao. IEEE Trans. on Aerospace \& Electronic Systems Vol.49(2013), p. $760-773$

[10] S. S. Chen, D. L. Donoho, M. A. Saunders. SIAM Journal on Scientific Computing Vol.20(1998), p. 33-61 